Título artículo / Títol article: Fast methodology for the reliable determination of nonylphenol in water samples by minimal labeling isotope dilution mass spectrometry

Autores / Autors

Revista:
Fabregat Cabello, Neus ; Castillo Tirado, Ángel ; Sanchis Llopis, Juan Vicente ; González Adelantado, Florenci Vicent ; Roig i Navarro, Antoni

Journal of Chromatography A Volume 1301, 2 August 2013

Preprint de l'autor

Cita bibliográfica / Cita bibliogràfica (ISO 690):

FABREGAT CABELLO, Neus, et al. Fast methodology for the reliable determination of nonylphenol in water samples by minimal labeling isotope dilution mass spectrometry. Journal of Chromatography A, 2013, 1301: 1926.

url Repositori UJI: 


\section{FAST METHODOLOGY FOR THE RELIABLE DETERMINATION OF NONYLPHENOL IN WATER SAMPLES BY MINIMAL LABELING ISOTOPE DILUTION MASS SPECTROMETRY ${ }^{1}$}

Neus Fabregat-Cabello ${ }^{a}$, Ángel Castillo ${ }^{a}$, Juan V. Sancho ${ }^{a}$, Florenci V. González ${ }^{b}$, Antoni Francesc Roig-Navarro*,a .

${ }^{a}$ Research Institute for Pesticides and Water, Universitat Jaume I, E-12071, Castellón, Spain.

${ }^{b}$ Departament de Química Inorgànica i Orgànica, Universitat Jaume I, E-12071, Castellón, Spain

*Corresponding Author: Tel: + 34964387359 ; fax: + 34964387368.

E-mail address: roig@uji.es (A.F. Roig-Navarro)

${ }^{1}$ Presented at the XII Scientific Meeting of the Spanish Society of Chromatography and Related Techniques, Tarragona, Spain, November 2012. 


\section{Abstract}

In this work we have developed and validated an accurate and fast methodology for the determination of 4-nonylphenol (technical mixture) in complex matrix water samples by UHPLC-ESI-MS/MS. The procedure is based on Isotope Dilution Mass Spectrometry (IDMS) in combination with Isotope Pattern Deconvolution (IPD), which provides the concentration of the analyte directly from the spiked sample without requiring any methodological calibration graph. To avoid any possible isotopic effect during the analytical procedure the in-house synthesized ${ }^{13} \mathrm{C}_{1}-4-(3,6$-dimethyl-3heptyl)phenol) was used as labeled compound. This proposed surrogate was able to compensate the matrix effect even from wastewater samples. A SPE preconcentration step together with exhaustive efforts to avoid contamination were included to reach the signal-to-noise ratio necessary to detect the endogenous concentrations present in environmental samples. Calculations were performed acquiring only three transitions, achieving limits of detection lower than $100 \mathrm{ng} / \mathrm{g}$ for all water matrix assayed. Recoveries within $83-108 \%$ and coefficients of variation ranging from $1.5 \%$ to $9 \%$ were obtained. On the contrary a considerable overestimation was obtained with the most usual classical calibration procedure using 4-n-nonylphenol as internal standard, demonstrating the suitability of the minimal labeling approach.

Keywords: Nonylphenol, Isotope dilution mass spectrometry, Minimal labeling, Isotope pattern deconvolution, LC-MS/MS, Wastewater analysis 


\section{Introduction}

Nonylphenols (NPs) are degradation products of non-ionic surfactants, NP polyethoxylates, which have been widely used in the production of detergents, plastics, textiles, paper and agricultural chemical products. Since they are able to mimic the structure of the natural hormone $17 \beta$-estradiol, which confers on them endocrine disrupting capabilities [1], NPs have been included in the list of priority substances in the Water Framework Directive (WFD) [2] and in the Directive of Environmental Quality Standards(EQSD) [3]. The new proposal for a Directive amending the WFD and EQSD (COM(2011)876) [4] legislates the mixture of isomers nonylphenol (CAS 25154-52-3) including isomers 4-nonylphenol (linear) (CAS 10440-5) and 4-nonylphenol (branched) (CAS 84852-15-3). Nevertheless, as far as we know the linear isomer has not been detected in water samples at significant concentrations and its estrogenic power is lower than in branched isomers [5]. Therefore, the evaluation of the harmful effects of NPs is focused on the determination of technical NP (NP), consisting mainly in a mixture of branched paraisomers $(>90 \%)[5,6]$.

Due to the ubiquitous presence of NP [1, 7] and the low levels required to assess the EQS for these compounds in a great variety of complicated matrices, the development of adequate analytical methods to determine NP is still a challenge [5, $6,8,9]$.

Both GC-MS [6, 8-10] equipped with a single quadrupole and LC-MS [11] with single quadrupole or LC-MS/MS [5, 7-9, 12-14] with a triple quadrupole (QqQ) have been widely used to quantify NP in environmental samples. Nevertheless, LC techniques are preferred over GC-MS because there is no need to perform any derivatization step, which increases the total analysis time and may show low yields in complex 
matrices [10]. On the other hand, in UHPLC-MS/MS the overall NP isomers elute as a single chromatographic peak, making possible the simultaneous quantification of NP with the same fragmentation pathway. Normally, an off-line preconcentration step by solid-phase extraction (SPE) is required to allow the determination of NP at the levels established in the legislation.

Nowadays there is still no consensus in the selection of a NP isomer which represents both the estrogenic power and the composition of NP in nature. Due to the complexity of the vast majority of environmental samples, the use of an internal standard (IS) during NP determination is almost mandatory. This selected IS should show the same behavior than NP, regarding sample treatment and instrumental measurement [5]. As far as we know, with the exception of Rabouan et al [5], all published papers have used some commercial technical mixture as quantification standard. On the other hand several internal standards have been employed. In GC/MS, isomers ${ }^{13} \mathrm{C}_{6}$-363-NP (4-(3,6-Dimethyl-3-heptyl)phenol-ring- $\left.{ }^{13} \mathrm{C}_{6}\right)$ [6] and 4sec-NP(4-(2,6-dimethylhept-3-yl)phenol)[10] have been used and each one was compared with the linear isomer 4-n-NP, other usual internal standard . Both papers consider the linear isomer unsuitable due to the different behavior during SPE step [6] or to the different derivatization yield [10] compared to branched NP. Regarding LC-MS (or MS/MS), 4-n-NP-d $d_{8}$ has been used satisfactorily by Loos et al [7, 12] although in older works [14] it has been considered as unsuitable due to low purity of the standard. Another deuterated isomer, 4-n-NP- $\mathrm{d}_{4}$, has been also employed as surrogate [13]. Ferguson et al. [11] discuss the potential limitations of the method using the isomer ${ }^{13} \mathrm{C}_{6}-4-n-N P$ as surrogate and 4-n-NP as internal standard. They conclude that the linear internal standard accounts for the matrix effect in the surrogate correctly since both isomers coelute. However, the branched NP elutes at 
different retention time, probably together with different matrix, and hence the quantification is approximate.

The use of an appropriated internal standard with identical retention time than the analyte might provide the true concentration of NP in complex matrix water samples. In this sense, according to González-Antuña et al. [15, 16] a minimal labeling (e.g. a single ${ }^{13} \mathrm{C}$ label in the molecule) ensures the same physicochemical behavior between the analyte and the isotopically labeled internal standard. The problem associated with this choice is the non-linear isotope dilution calibration graphs owing to the spectral overlap. Nevertheless it can be overcome using Isotope Pattern Deconvolution (IPD).

IPD permits the calculation of the molar fraction of natural and labeled compound in the spiked sample by multiple linear regression using the whole or a part of the mass isotopomer distribution [17]. In addition, this alternative approach does not require any methodological calibration graph, so the total analysis time is drastically reduced. Recently, IPD has been adapted to the determination of diclofenac by UHPLCMS/MS, demonstrating its applicability to tandem mass spectrometry [18]. In this work, we propose a procedure based on minimal labeling and IPD for the determination of NP in water samples by SPE-UHPLC-MS/MS. For this purpose, the labeled branched isomer ${ }^{13} \mathrm{C}_{1}-4-\left(3,6\right.$-dimethyl-3-heptyl)phenol $\left({ }^{13} \mathrm{C}_{1}-363-\mathrm{NP}\right)$ has been synthesized in our laboratory and characterized in terms of isotope composition and concentration. Furthermore, possible matrix effect has been corrected since labeled NP surrogate, enriched in a single carbon atom, coelutes with NP. The method has been validated in bottled water, effluent wastewater and influent wastewater spiked at two concentration levels. Finally, the figures of merit provided 
by the developed methodology were compared with those obtained by the usual external calibration using 4-n-NP as internal standard.

\section{Materials and Methods}

\subsection{Reagents and materials}

The technical 4-nonylphenol mixture (NP) of chain isomers (no. 290858) was purchased from Sigma-Aldrich (Steinheim, Germany) and 4-n-nonylphenol (4-n-NP) were delivered by Dr. Ehrenstorfer (Augsburg, Germany). Stock solutions were prepared by dissolving the corresponding standards in dichloromethane. All stock solutions were stored at $-20^{\circ} \mathrm{C}$ and employed to prepare daily gravimetrically diluted working standard solutions in methanol. Methanol, acetonitrile and dichloromethane solvents (analysis grade) as well as formic acid (reagent grade) and ammonium acetate (reagent grade) were provided by Scharlau (Barcelona, Spain). Solid phase extraction (SPE) was performed using $\mathrm{C} 18$ cartridges Extrabond of $3 \mathrm{~mL}$ volume and $500 \mathrm{mg}$ sorbent which were also provided by Scharlau. The $\mathrm{pH}$ of the mobile phase was adjusted approximately to 7 by adding ammonium hydroxide from Fluka (Buchs, Switzerland). HPLC-grade water was obtained by purifying demineralized water in a Milli-Q gradient A10 (Millipore, Bedford, MA, USA). Commercially bottled water stored in polyethylene terephthalate (PET) bottles was employed.

For the synthesis of 4-(3,6-dimethyl-3-heptyl) phenol, boron trifluoride $\left(\mathrm{BF}_{3}\right)$ diethyl etherate, n-hexane, 3,6-dimethyl-3-heptanol and phenol were purchased from Alfa Aesar (Karlsruhe, Germany). The ${ }^{13} \mathrm{C}_{1}-4$-(3,6-dimethyl-3-heptyl)phenol $\left({ }^{13} \mathrm{C}_{1}-\mathrm{NP}\right)$ was synthesized using ${ }^{13} \mathrm{C}_{1}$-phenol from Cambridge Isotope Laboratories (Andover, MA, USA). 


\subsection{Synthesis of ${ }^{13} C_{1}$-nonylphenol}

The synthesis of ${ }^{13} \mathrm{C}_{1}-4$ (3,6-dimethyl-3-heptyl)-phenol ( or ${ }^{13} \mathrm{C}_{1}-363 \mathrm{NP}$ ) was based on a Friedel-Crafts alkylation of ${ }^{13} \mathrm{C}_{1}$-labelled phenol and a tertiary nonylalcohol employing $\mathrm{BF}_{3}$-ether complex as catalyst $[19,20]$. The procedure is illustrated in Figure 1. As can be observed, the selected labeling position corresponds to the carbon linked to the hydroxyl group, which remains in the main fragment ions measured by tandem mass spectrometry.

Experimental procedure for the preparation of ${ }^{13} \mathrm{C}_{1}-4(3,6$-dimethyl-3-heptyl)-phenol: To a stirred solution of ${ }^{13} \mathrm{C}_{1}$-labelled phenol (125 mg, $\left.1.31 \mathrm{mmol}\right)$ and 3,6-dimethyl-3heptanol (126.5 mg, $0.88 \mathrm{mmol})$ in hexanes $(50 \mathrm{~mL})$ was added boron trifluoride etherate $(156 \mu \mathrm{L}, 0.9 \mathrm{mmol})$. The resulting mixture was stirred overnight, then icewater $(50 \mathrm{~mL})$ was added and extracted with hexanes $(3 \times 30 \mathrm{~mL})$. The organic layers were dried (sodium sulfate), filtered and concentrated under vacuum to afford a a crude oily product which was purified through liquid chromatography (silica-gel, hexanes: ethyl acetate $(15: 1))$ to afford $140 \mathrm{mg}$ (yield $=72 \%$ ) and characterized by ${ }^{1} \mathrm{H}$ NMR and ${ }^{13} \mathrm{C}$ NMR.

Spectroscopic data of ${ }^{13} \mathrm{C}_{1}-4$ (3,6-dimethyl-3-heptyl)-phenol: ${ }^{1} \mathrm{H}$ NMR $\left(\mathrm{CDCl}_{3}, 500\right.$ $\mathrm{MHz}) \delta 7.16(1 \mathrm{H}, \mathrm{d}, J=9.2 \mathrm{~Hz}), 7.14(1 \mathrm{H}, \mathrm{d}, J=9.1 \mathrm{~Hz}), 6.81(1 \mathrm{H}, \mathrm{d}, J=8.8 \mathrm{~Hz})$, $6.80(1 \mathrm{H}, \mathrm{d}, J=8.8 \mathrm{~Hz}), 5.40(1 \mathrm{H}, \mathrm{br} \mathrm{s}), 1.74-1.64(2 \mathrm{H}, \mathrm{m}), 1.59-1.39(\mathrm{~m}, 3 \mathrm{H}), 1.24$ $(3 \mathrm{H}, \mathrm{s}), 1.08-1.01(1 \mathrm{H}, \mathrm{m}), 0.92-0.85(1 \mathrm{H}, \mathrm{m}), 0.85(6 \mathrm{H}, \mathrm{t}, J=6.9 \mathrm{~Hz}), 0.69(3 \mathrm{H}, \mathrm{t}, J$ $=7.5 \mathrm{~Hz}) \mathrm{ppm} ;{ }^{13} \mathrm{C} \mathrm{NMR}\left(\mathrm{CDCl}_{3}, 125 \mathrm{MHz}\right) \delta 152.8,140.3(\mathrm{~d}, J=8.2 \mathrm{~Hz}), 127.6$, $114.8(\mathrm{~d}, J=66.2 \mathrm{~Hz}), 40.6,40.3,35.7,33.2,28.7,23.6,22.7,8.7 \mathrm{ppm}$.

Purity was evaluated by GC-EI-MS (see Figure 2). Since only one peak was obtained, the content of ${ }^{13} \mathrm{C}_{1}$ - nonylphenol was established as higher than $99.9 \%$. The mass spectra of the ${ }^{13} \mathrm{C}_{1}$ - nonylphenol and the analogous natural analogue are 
also included in Figure 2. As can be observed, the main fragment ions corresponding to the labelled compound are shifted by one mass unit.

\subsection{Instrumentation}

Identification and quantification of analytes were carried out using an Acquity UPLC system (Waters Corp., Milford, MA, USA), equipped with a binary solvent manager and sample manager. An Acquity UPLC HSS T3 column $1.8 \mu \mathrm{m}, 2.1 \mathrm{~mm} \times 100 \mathrm{~mm}$ (i.d.) (Waters) was used. The column oven was set at $40^{\circ} \mathrm{C}$, the flow rate was 300 $\mu \mathrm{L} / \mathrm{min}$ and the injection volume was $20 \mu \mathrm{L}$. The separation was performed under isocratic conditions with a mobile phase consisted of $95 \%$ methanol/bottled water with $0.01 \%$ ammonia and $0.1 \mathrm{mM}$ ammonium acetate.

A TQD (quadrupole-hexapole-quadrupole) tandem mass spectrometer with an orthogonal Z-spray-electrospray interface (Waters) was used for UHPLC analysis. The mass spectrometer was operated in negative mode electrospray ionization in selected reaction monitoring (SRM) mode. Drying as well as nebulising gas was nitrogen, obtained from a nitrogen generator $\mathrm{N}_{2}$ LC-MS adapted forLC-MS analysers (Claind, Teknokroma, Barcelona, Spain). The operating parameters of the spectrometer were capillary voltage $3.30 \mathrm{kV}$, and source and desolvation temperatures 120 and $350{ }^{\circ} \mathrm{C}$, respectively. The cone gas and desolvation flow were set at 40 and $400 \mathrm{~L} / \mathrm{h}$. For operation in MS/MS mode, collision gas was Argon $99.995 \%$ (Praxair, Madrid, Spain) with a pressure of approximately $4 \cdot 10^{-3} \mathrm{mbar}$ in the collision cell. Dwell times of $0.1 \mathrm{~s} / \mathrm{scan}$ were chosen. Masslynx v 4.1 (Waters, Manchester, UK) software was used to process the quantitative data obtained. Gas chromatography analyses were performed using an Agilent 6890 Series GC equipped with a split-splitless injection port, and mass spectrometric detector 
(Agilent 5973 N MSD) with an electron ionization (EI) source and MPS2 autosampler from Gerstel (Linthicum, MD, USA). The GC was fitted with a $30 \mathrm{~m} \times 0.25$-mm (i.d.), 0.25- $\mu \mathrm{m}$ DB-5MS column (Hewlett-Packard). The column temperature was initially held at $60^{\circ} \mathrm{C}$ for $1 \mathrm{~min}$, and then a temperature ramp of $10^{\circ} \mathrm{C} / \mathrm{min}$ was applied until $300{ }^{\circ} \mathrm{C}$ and this temperature was maintained for $1 \mathrm{~min}$. Helium was used as carrier gas at a flow rate of $1 \mathrm{~mL} / \mathrm{min}$. Temperature of the injector was set to $280^{\circ} \mathrm{C}$ and a sample volume of $2 \mu \mathrm{L}$ was injected in splitless mode.

\subsection{Extraction of technical NP from water}

Before loading the SPE cartridges, all effluent (EWW) and influent (IWW) wastewaters with observable suspended particulate matter were decanted by pouring the water after sedimentation slowly from the sample bottles into clean $1 \mathrm{~L}$ glass (Schott-Duran) bottles [12]. IWW samples were diluted five times in bottled water due to their high complexity and organic matter content. C18 cartridges were previously rinsed with $6 \mathrm{~mL}$ of acetone and conditioned with $6 \mathrm{~mL}$ of methanol and $6 \mathrm{~mL}$ of bottled water. $200 \mathrm{~mL}$ of water were spiked with an appropriate amount of the ${ }^{13} \mathrm{C}_{1^{-}}$ $\mathrm{NP}$, according to the random error propagation theory [21]. Accurate and precise results will be obtained when the ratio of concentrations between the natural and labeled compound is in the range of 0.1 to 10 . To prevent volumetric errors, the amount of sample and spike added were determined gravimetrically. Then, the sample was passed through the cartridge by gravity (flow around $3 \mathrm{~mL} / \mathrm{min}$ ). After drying for $30 \mathrm{~min}$, analytes were eluted with $5 \mathrm{~mL}$ of methanol. Finally, the extract was evaporated to a volume of around $0.5 \mathrm{~mL}$ with a gentle stream of nitrogen (40 ${ }^{\circ} \mathrm{C}$ ) in a water bath. 


\subsection{Reduction of blank contamination}

One of the main challenges in the determination of nonylphenol is having under control all the possible contamination sources [22]. The amount of NP coming from all the possible contamination sources of the developed method has been minimized and subtracted to the total measured NP concentration. The main contribution of the sought compound was from Milli-Q water, LC septa and SPE cartridges. Actually, due to the noticeable levels detected in Milli-Q water, bottled water stored in PET bottles has been employed as a sample blank. The selection of appropriate LC septa

is also critical, since most PTFE/Silicone septa in LC vials filled with the methanolic extract of sample releases NP. To prevent any possible contamination LC vials and screw caps were rinsed with methanol. On the other hand, SPE cartridges were rinsed with acetone and dried before use.

Finally, according to the cleaning procedure of Chen et al. [23], glassware was washed with tap water, sonicated with Milli-Q water and then rinsed with acetone, dichloromethane and methanol. Plastic material (e.g. SPE adapters) were washed with tap water and rinsed with acetone and methanol.

After applying all the cleaning procedure, reagent blanks obtained were within the range of $10-30 \mathrm{pg} / \mathrm{g}$.

\subsection{Determination of technical NP by Isotope Pattern Deconvolution}

For the quantification of NP three transitions were employed: $219>133,220>134$ and $221>135$. The molar fractions $\left(X_{n a t}\right.$ and $\left.X_{l a b}\right)$ of natural abundance and labeled NP were calculated by multiple linear regression using the following system of equations: 


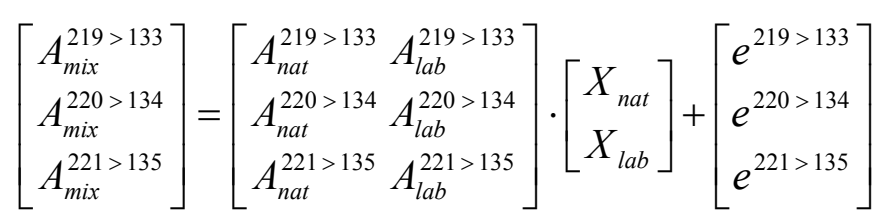

where the $A_{m i x}^{i>j}$ values corresponds to the measured relative abundance of the compound in the mixture for the SRM transition of nominal masses $\mathrm{i}>\mathrm{j}$ and the $A_{\text {nat }}^{i>j}$ and $A_{l a b}^{i>j}$ values the theoretically calculated or experimentally measured abundances for the natural NP and labeled ${ }^{13} \mathrm{C}_{1}-\mathrm{NP}$, respectively. The amount (mols) of natural abundance NP in the sample, $N_{\text {nat }}$, is then calculated using equation (2) where the amount (mols) of labeled NP added, $N_{l a b}$, must be known.

$$
N_{\text {nat }}=N_{\text {lab }} \frac{X_{\text {nat }}}{X_{\text {lab }}}
$$

Confirmation of positive findings was carried out by calculating the peak area ratios between the quantification transition (Q) $219>133$ and the confirmation transition (q) $219>147$, and comparing them with ion-ratios from a reference standard.

\section{Results and discussion}

As it was reported in a previous work [18], the application of IPD to tandem mass spectrometry ( $Q q Q)$ in Selected Reaction Monitoring (SRM) mode requires the calculation of the product ions mass isotopomer distribution of natural abundance and isotope-labeled compound. For the quantification of NP the neutral loss of a $\mathrm{C}_{6} \mathrm{H}_{14}$ group in the molecule was selected [9]. The resulting mass isotopomer distribution coming from each isotope pattern were computed using the software 
Isopatrn implemented by L. Ramaley et al. [24]. Then, molar balances for all these transitions were established as a function of each compound. The procedure is illustrated in Figure 3, where the measured isotope pattern of the spiked sample is deconvoluted into its constituting components by multiple least squares, providing the molar fraction of NP $\left(X_{\text {nat }}\right)$ and ${ }^{13} \mathrm{C}_{1}-\mathrm{NP}\left(X_{\text {lab }}\right)$.

\subsection{Study of the spectral purity and isotope enrichment of ${ }^{13} C_{1}$-nonylphenol}

The accuracy of the IPD procedure relies on the correct value of the relative abundances employed for NP and ${ }^{13} \mathrm{C}_{1}-\mathrm{NP}$. In tandem mass spectrometry different fragmentation pathways can take place simultaneously, leading to different product ions. The overlapping of the selected fragmentation cluster with any adjacent cluster ion (with one extra hydrogen or one hydrogen less) [15] modifies the isotopomer distribution of each isotope pattern, thus it has to be taken into account. In order to quantify the contribution of each individual source to the overall selected cluster, a complete cluster characterization was performed before isotope dilution analysis using multiple linear regression [15, 25]. A standard solution of around $500 \mathrm{ng} / \mathrm{g}$ of NP in methanol was injected 10 times to the UHPLC-MS/MS in SRM mode, including all transitions corresponding to the fragment clusters $\left[\mathrm{M}-\mathrm{H}-\mathrm{C}_{6} \mathrm{H}_{14}\right]^{-},\left[\mathrm{M}-\mathrm{H}-\mathrm{C}_{6} \mathrm{H}_{15}\right]^{-}$and $\left[\mathrm{M}-\mathrm{H}-\mathrm{C}_{6} \mathrm{H}_{13}\right]^{-}$. The results showed a contribution of $96.0 \pm 0.3$ for $\left[\mathrm{M}-\mathrm{H}-\mathrm{C}_{6} \mathrm{H}_{14}\right]^{-}$and 4.0 $\pm 0.3 \%$ for $\left[\mathrm{M}-\mathrm{H}-\mathrm{C}_{6} \mathrm{H}_{15}\right]^{-}$cluster. Additionally, spectral purity was evaluated in bottled water, surface water and effluent and influent wastewater. As it can be seen in Table 1 , the fragmentation factors did not change with the matrix of the sample. Consequently, the theoretical isotopomer distribution of the natural abundance nonylphenol was recalculated to include the $\left[\mathrm{M}-\mathrm{H}-\mathrm{C}_{6} \mathrm{H}_{15}\right]^{-}$contribution. 
For the calculation of the isotopomer distribution of ${ }^{13} \mathrm{C}_{1}-\mathrm{NP}$, the extent of labeling must be determined previously. To this end, standard solutions containing $500 \mathrm{ng} / \mathrm{g}$ of ${ }^{13} \mathrm{C}_{1}$-NP were injected in the UHPLC-MS/MS system $(n=5)$. The measured cluster abundances were compared with those calculated for theoretical enrichments of the labeled compound from 96.5 to $99.5 \%{ }^{13} \mathrm{C}_{1}$. It is worth stressing that the fragmentation pattern is independent of the isotope composition of the molecule (natural or labeled), thus the theoretical cluster abundances of ${ }^{13} \mathrm{C}_{1}$-NP were also recalculated including the $\left[\mathrm{M}-\mathrm{H}-\mathrm{C}_{6} \mathrm{H}_{15}\right]^{-}$contribution. Since the lower the sum of squares of errors, the more similar the mass isotopomer distribution examined, the computation of such errors allowed us to find the ${ }^{13} \mathrm{C}_{1}$ enrichment of the molecule. A clear minimum was obtained for around 98 atom $\%{ }^{13} \mathrm{C}_{1}$ for all the replicates (see Figure 4). Accordingly, the theoretical isotopomer distribution of the labeled compound was calculated assuming a ${ }^{13} \mathrm{C}_{1}$ enrichment of $98 \%$.

Once obtained the whole isotope distribution of NP and ${ }^{13} \mathrm{C}_{1}-\mathrm{NP}$, the IPD approach can be applied. The resulting overdetermined system of equations can be expressed, in matrix form, as

$$
\left[\begin{array}{l}
A_{m i x}^{219>133} \\
A_{m i x}^{220>133} \\
A_{m i x}^{220>134} \\
A_{m i x}^{221>133} \\
A_{m i x}^{221>134} \\
A_{m i x}^{221>135} \\
A_{m i x}^{222>133} \\
A_{m i x}^{222>134} \\
A_{m i x}^{222>135} \\
A_{m i x}^{222>136}
\end{array}\right]=\left[\begin{array}{ll}
0.81280 & 0.01643 \\
0.05740 & 0.03470 \\
0.08027 & 0.80661 \\
0.00173 & 0.00227 \\
0.00555 & 0.05659 \\
0.00521 & 0.07090 \\
0.00003 & 0.00006 \\
0.00016 & 0.00170 \\
0.00036 & 0.00490 \\
0.00026 & 0.00440
\end{array}\right] \cdot\left[\begin{array}{l}
X_{\text {nat }} \\
X_{l a b}
\end{array}\right]+\left[\begin{array}{l}
e^{219>133} \\
e^{220>133} \\
e^{220>134} \\
e^{221>133} \\
e^{221>134} \\
e^{221>135} \\
e^{222>133} \\
e^{222>134} \\
e^{222>135} \\
e^{222>136}
\end{array}\right]
$$


The $A_{\text {mix }}^{i>j}$ values corresponds to the measured relative abundance of the compound in the mixture for the SRM transition of nominal masses $\mathrm{i}>\mathrm{j}$. These values are determined experimentally by peak area integration of several product ions. The relative abundance of each fragment is then calculated dividing the peak area of a given SRM transition by the sum of all areas. As we have more parameters (transitions) than unknowns (molar fractions) an error vector is included in eqn (3). The best values of $X_{n a t}$ and $X_{l a b}$ are found by least square minimization of the error vector 'e'.

Finally, only three SRM transitions were selected $(219>133,220>134$ and $221>$ 135), according to the random error propagation theory, to make possible the application of this alternative technique in routine multi-residue analysis.

\subsection{Optimization of chromatographic conditions}

In order to achieve the elution of NP as well as 4-n-NP different mobile phases based on methanol or acetonitrile and water were tested. The addition of different modifiers such as ammonia, ammonium acetate and formic acid were also evaluated. The use of gradient conditions led to inappropriate higher areas due to the trace nonylphenol contamination of Milli-Q water, which is accumulated at the head of the column. As a consequence, isocratic conditions were employed. Regarding mobile phase composition, higher sensitivity was observed when the analyte was eluted with methanol instead of acetonitrile. The addition of ammonia as a modifier also increased the signal of the analyte while formic acid decreased it. We also observed that combination of ammonium acetate with ammonia did not decrease significantly the signal but stabilized it. According to this, a mobile phase consisted in 95:5 methanol:water containing $0.01 \%$ ammonia and $0.1 \mathrm{mM}$ ammonium acetate was 
selected. In this conditions the compounds were eluted in a 3-minutes run without the need of column stabilization between injections. Finally, we reduced the background associated to blank contamination using bottled water instead of Milli-Q water.

\subsection{Solid-phase extraction}

To reach the sensitivity necessary to detect the typical concentrations of NP present in environmental samples an off-line pre-concentration step was included. For this purpose, bottled water was spiked with NP at two concentration levels; namely, 0.1 $\mathrm{ng} / \mathrm{g}$ and $1 \mathrm{ng} / \mathrm{g}$.

In spite of some authors reject the use of any plastic material during the analytical procedure [22] SPE was selected because it is a simple and rapid isolation technique widely used in routine laboratories. The use of Oasis HLB cartridges was rejected due to the low recoveries described in literature $[26,27]$ associated to the high hydrophobicity of NP. Following literature recommendations [26], samples were passed through C18 cartridges. The elution solvents tested were acetonitrile and methanol. Although no relevant differences were noticed, the mobile phase is based on the last one; so, methanol was selected for elution (5 mL).

Once the type of cartridge and elution solvent were selected, different sample volumes were evaluated. It was observed that $100 \mathrm{~mL}$ of water spiked at the low concentration tested were not enough to confirm the presence of nonylphenol, i.e. the peak area corresponding to the transition $219>147$ was too low. Therefore, the sample volume was increased up to $200 \mathrm{~mL}$, which did not exceed the breakthrough volume. In these conditions, the SPE recoveries were around $70 \%$. In any case, the

use of ${ }^{13} \mathrm{C}_{1}$-NP as surrogate allowed us to obtain satisfactory recoveries (see next section). 


\subsection{Method validation}

Analytical characteristics of the developed method were evaluated in three types of water samples (bottled, EWW and IWW). Accuracy and precision were estimated from recovery experiments of the target compound at two concentration levels, analyzing three independent replicates injected three times each. Bottled samples and EWW were spiked at 0.1 and $1 \mathrm{ng} / \mathrm{g}$ of NP, while IWW was spiked at 1 and 10 $\mathrm{ng} / \mathrm{g}$ because of its higher endogenous nonylphenol concentration. Afterwards, the synthesized labeled compound ${ }^{13} \mathrm{C}_{1}$-NP was added at the same concentration level as NP. Since it was not possible to obtain a true blank sample, bottled water, EWW and IWW were previously analyzed and the concentrations of the analyte found were subtracted from the spiked samples, resulting in higher errors in the recovery calculation. Figure 5 shows the chromatograms corresponding to the different spiked water samples analyzed at their low validation level. As can be seen, the labeled and natural nonylphenol co-elute, thus there were no chromatographic isotopic effects and recoveries within $83-108 \%$ were obtained (Table 2). This values indicate that matrix effect is correctly accounted for. Precision, expressed as repeatability in terms of relative standard deviation (RSD), was from 1.5 to $9 \%$, which are considerably lower than the maximum required by SANCO guide [28] (RSD $\leq 20 \%$ ). The spiked samples were also quantified by classical calibration curve employing 4$\mathrm{n}-\mathrm{NP}$ as internal standard. To this end, increasing concentrations $(0.05-5 \mu \mathrm{g} / \mathrm{g})$ of NP in methanol containing $0.4 \mu \mathrm{g} / \mathrm{g}$ of $4-\mathrm{n}-\mathrm{NP}$ were injected in triplicate through the UHPLC- MS/MS. To build the calibration graph, relative areas were used, selecting the transition $(219>106)$ for the linear nonylphenol and $(219>133)$ for NP. We spiked samples at $1 \mathrm{ng} / \mathrm{g}$ of 4-n-NP and extracted them as described previously. As 
can be seen in Table 2, a clear overestimation was observed when using such internal standard, especially in effluent wastewater. Due to the fact that 4-n-NP elutes at different retention time than NP it cannot mimic the behavior of the analyte, especially in complicated sample matrix such as effluent wastewater in which no sample dilution was performed. Contrary to previous publications [14], we observed that in this case linear nonylphenol was not well suited as internal standard. In the line of European Commission Decision 2002/657/EC [29], the confirmation of the positive findings were carried out by calculating the peak area ratio between the most intense transition $(219>133)$ and the confirmation transition $(219>147)$. In spite of the theoretical contribution of ${ }^{13} \mathrm{C}_{1}-\mathrm{NP}$ to these transitions, this was low enough to not to induce false positives, making possible the appropriate confirmation of the samples.

Finally, the limit of detection (LOD) and limit of quantification (LOQ) were estimated for $S / N=3$ and $S / N=10$, respectively, from the chromatogram of the lowest concentration level tested. The values obtained are shown in Table 3 and were similar to other conventional methods proposed for the determination of NP $[14,26$, 30]. In the line of the new proposal for a Water Framework Directive, the annual average value for technical nonylphenol in waters is $0.3 \mu \mathrm{g} / \mathrm{L}$, whereas the maximum acceptable concentration (MAC-EQS) is $2 \mu \mathrm{g} / \mathrm{L}$. Thus the developed procedure could be very useful to carry out a fast determination of NP at the levels established by the legislation.

\section{Conclusions}

An UHPLC-MS/MS method for the determination of NP in wastewater samples has been developed and validated. The selected quantification procedure is based on 
isotope dilution mass spectrometry and isotope pattern deconvolution which provides reliable and fast results. Each sample is spiked with a minimal labeled internal standard, separated in the UHPLC-MS/MS system and quantified without the need to resort to any methodological calibration graph. The labeled branched isomer ${ }^{13} \mathrm{C}_{1}-4-$ (3,6-dimethyl-3-heptyl)phenol has been synthesized and used as surrogate. Since this compound is enriched in a single carbon atom it shows identical physicochemical properties than the technical NP mixture assayed and therefore the same retention time. Thus, it provides a suitable matrix effect correction in ESI ionization source. The validated method is robust and sensitive, even in influent wastewater. High accuracy (recovery near 100\%) and precision (CV bellow 10\%) are obtained which confer on the procedure high reliability for the determination of NP at the EU legislation requirements. On the contrary, when quantifying with the most usual classical calibration procedure with 4-n-NP as internal standard important overestimation was observed, especially in effluent wastewater.

Future works will be in line with the synthesis of new single ${ }^{13} \mathrm{C}$ labeled standards for the development of multiresidue methods with the inclusion of other legislated alkylphenols combining IPD calculations. Moreover, alternative extraction methods able to be used in routine laboratories should be investigated to reduce the time associated to the off-line preconcentration step by SPE.

\section{Acknowledgement}

The authors acknowledge the financial support from Generalitat Valenciana (Research group of excellence Prometeo 2009/054). N.Fabregat-Cabello also acknowledges the Generalitat Valenciana for her Ph.D. research grant under the Program VALi+D. We would like to thank S.Ballester and J.L. Aranda (IPROMA) for 
their useful comments and help. Finally the authors are very grateful to the Serveis

Centrals d'Instrumentació Científica (SCIC) of University Jaume I for using Acquity and TQD instruments.

\section{References}

[1] A. Soares, B. Guieysse, B. Jefferson, E. Cartmell, J.N. Lester, Environ. Int., 34 (2008) $1033-49$.

[2] Directive 2000/60/EC, Establishment and Framework for Community Action in the Field of Water Policy, European Parliament and the Council of the European Union, 2000.

[3] Directive 2008/105/EC, Environmental Quality Standards in the Field of Water Policy, Amending and Subsequently Repealing Council Directives 82/176/EEC, 83/513/EEC, 84/156/EEC, 84/491/EEC, 86/280/EEC and Amending Directive 2000/60/EC, European Parliament a, 2008.

[4] Proposal for a Directive of the european parliament and of the council amending Directives 2000/60/EC and 2008/105/EC as regards priority substances in the field of water policy (COM(2011)876), 2012.

[5] S. Rabouan, A. Dupuis, A. Cariot, M. Albouy-Llaty, V. Migeot, B. Legube, Trend. Anal. Chem., 37 (2012) 112-123.

[6] E. Stottmeister, O.P. Heemken, P. Hendel, G. Donnevert, S. Frey, H. Allmendinger, G. Sawal, B. Jandel, S. Geiss, R. Donau, A. Koch, I. Heinz, M. Ottaviani, E. Veschetti, W. Hartl, C. Kubwabo, C. Benthe, V. Tobinski, H. Woldmann, R. Spilker, Anal. Chem., 81 (2009) 6765-6773.

[7] R. Loos, B.M. Gawlik, G. Locoro, E. Rimaviciute, S. Contini, G. Bidoglio, Environ. Pollut., 157 (2009) 561-8.

[8] E. Olkowska, Ż. Polkowska, J. Namieśnik, Talanta, 88 (2012) 1-13.

[9] E. Gallart-Ayala, E. Moyano, M.T. Galceran, Mass. Spectrom. Rev., (2010) 776-805.

[10] A.R. Fischer, N.T.P. Lan, C. Wiedemann, P. Heide, P. Werner, A.W. Schmidt, G. Theumer, H.-J. Knölker, J. Chromatogr. A., 1217 (2010) 2950-2955.

[11] P.L. Ferguson, C.R. Iden, B.J. Brownawell, Anal. Chem., 72 (2000) 4322-4330.

[12] R. Loos, G. Locoro, S. Contini, Water Res., 44 (2010) 2325-35.

[13] N. Salgueiro-González, E. Concha-Graña, I. Turnes-Carou, S. Muniategui-Lorenzo, P. López-Mahía, D. Prada-Rodríguez, J. Chromatogr. A., 1223 (2012) 1-8. 
[14] R. Loos, G. Hanke, G. Umlauf, S.J. Eisenreich, Chemosphere, 66 (2007) 690-699.

[15] A. González-Antuña, P. Rodríguez-González, G. Centineo, J.I. García Alonso, Analyst, 135 (2010) 953-964.

[16] A. González-Antuña, P. Rodríguez-González, I. Lavandera, G. Centineo, V. Gotor, J.I. García Alonso, Anal. Bioanal. Chem., 402 (2012) 1879-1888.

[17] P. Rodríguez-González, J.I. García Alonso, J. Anal. Atom. Spectrom., 25 (2010) 239.

[18] Á. Castillo, E. Gracia-Lor, A.F. Roig-Navarro, J.V. Sancho, P. Rodríguez-González, J.I.G. Alonso, Anal. Chim. Acta (2013). http://dx.doi.org/10.1016/j.aca.2012.11.033.

[19] R. Vinken, B. Schmidt, A. Schäffer, J. Labelled Compd. Rad., 45 (2002) 1253-1263.

[20] T. Uchiyama, M. Makino, H. Saito, T. Katase, Y. Fujimoto, Chemosphere, 73 (2008) S60-S65.

[21] J.I. García Alonso, Anal. Chim. Acta, 312 (1995) 57-78.

[22] N. Salgueiro-González, E. Concha-Graña, I. Turnes-Carou, S. Muniategui-Lorenzo, P. López-Mahía, D. Prada-Rodríguez, Talanta, 101 (2012) 413-419.

[23] W.L. Chen, G.S. Wang, J.C. Gwo, C.Y. Chen, Talanta, 89 (2012) 237-45.

[24] L. Ramaley, L. Cubero Herrera, Rapid Commun. Mass Sp., (2008) 2707-2714.

[25] J. Meija, J. a Caruso, J. Am. Soc. Mass Spectr., 15 (2004) 654-8.

[26] T. Vega-Morales, Z. Sosa-Ferrera, J.J. Santana-Rodríguez, J. Hazard. Mater., 183 (2010) $701-711$.

[27] N. Fontanals, R.M. Marcé, F. Borrull, J. Chromatogr. A., 1152 (2007) 14-31.

[28] European Commission, DG-SANCO, Method validation and quality control procedures for pesticide residues analysis in food and feed, Document No. SANCO/12495/2011, Brussels, December, 2011. http://ec.europa.eu/food/plant/protection/pesticides/docs/qualcont, 2012.

[29] European Union Decision 2002/657/EC, Off. J. Eur. Commun., L221, 2002, pp. 8-36 (12 August 2002), .

[30] M. Petrovic, A. Diaz, F. Ventura, D. Barceló, Anal. Chem., 73 (2001) 5886-95. 


\section{FIGURE 1}

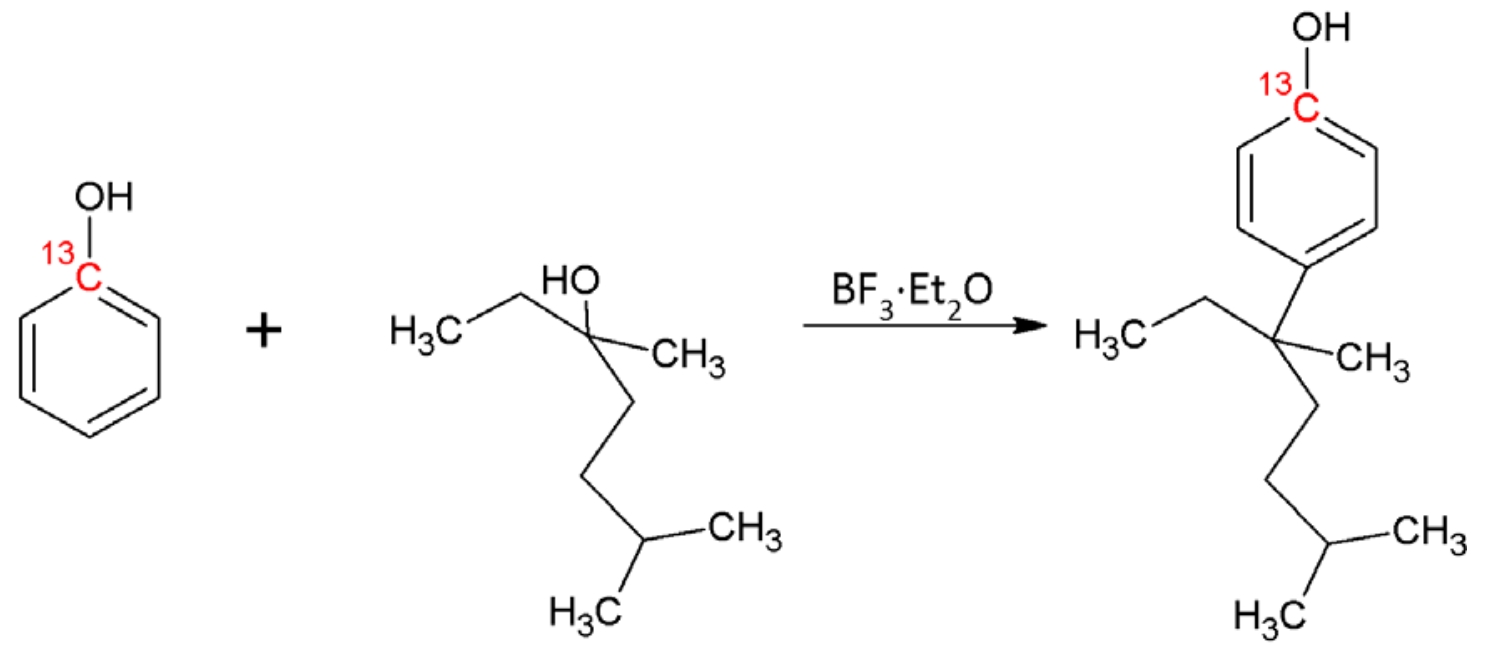

Figure 1. Synthesis pathway of labeled ${ }^{13} \mathrm{C}_{1}-4$-(3,6-Dimethyl-3-heptyl)-phenol $\left({ }^{13} \mathrm{C}_{1}-\mathrm{NP}\right)$. 


\section{FIGURE 2}

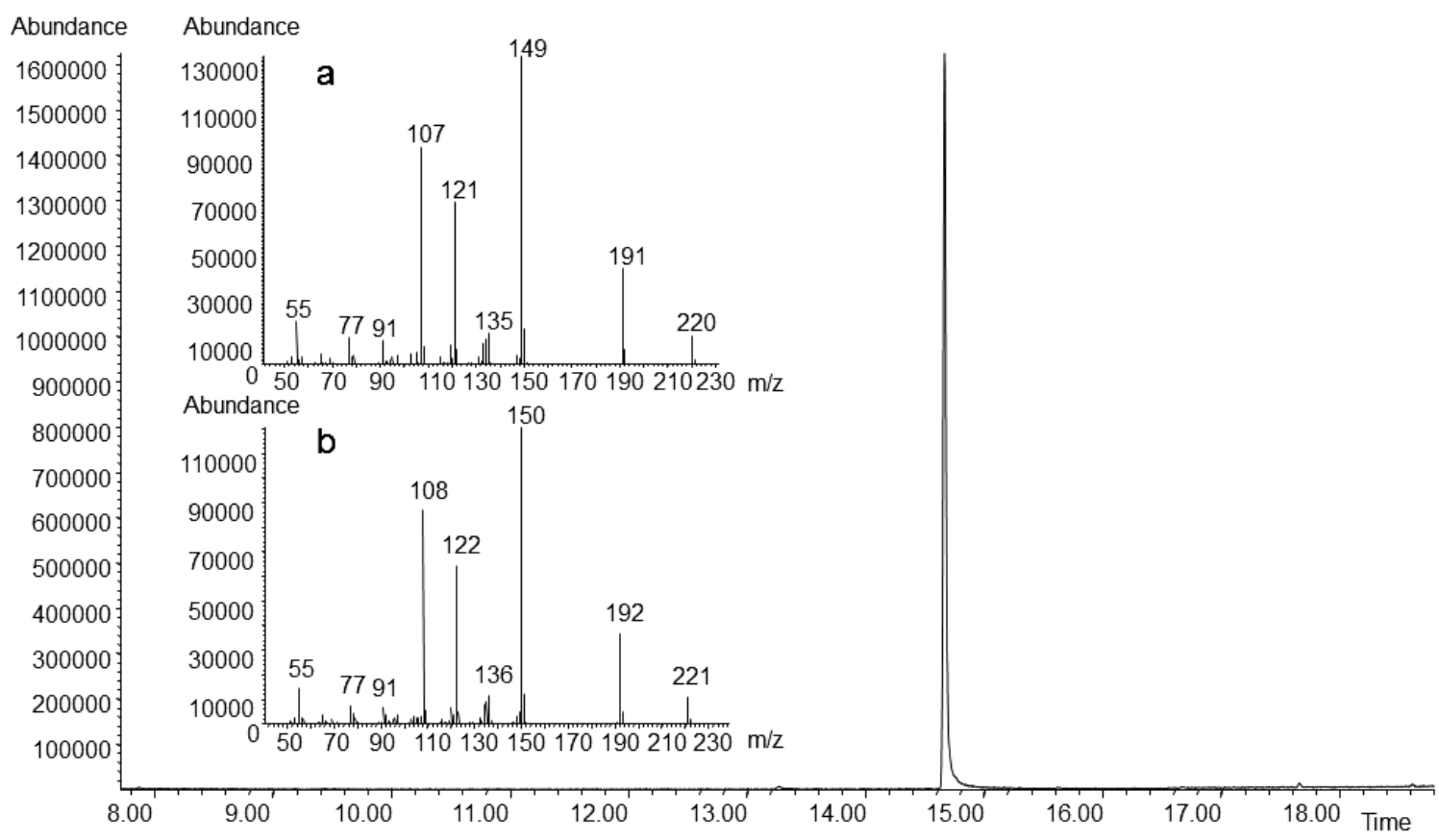

Figure 2. GC-El-MS chromatogram of ${ }^{13} \mathrm{C}_{1}-4$-(3,6-dimethyl-3-heptyl)phenol and mass spectra of a standard of a 4-(3,6-dimethyl-3-heptyl)phenol and $\mathbf{b}^{13} \mathrm{C}_{1}-4$-(3,6-dimethyl-3-heptyl)phenol $\left({ }^{13} \mathrm{C}_{1}\right.$ - NP). 


\section{FIGURE 3}

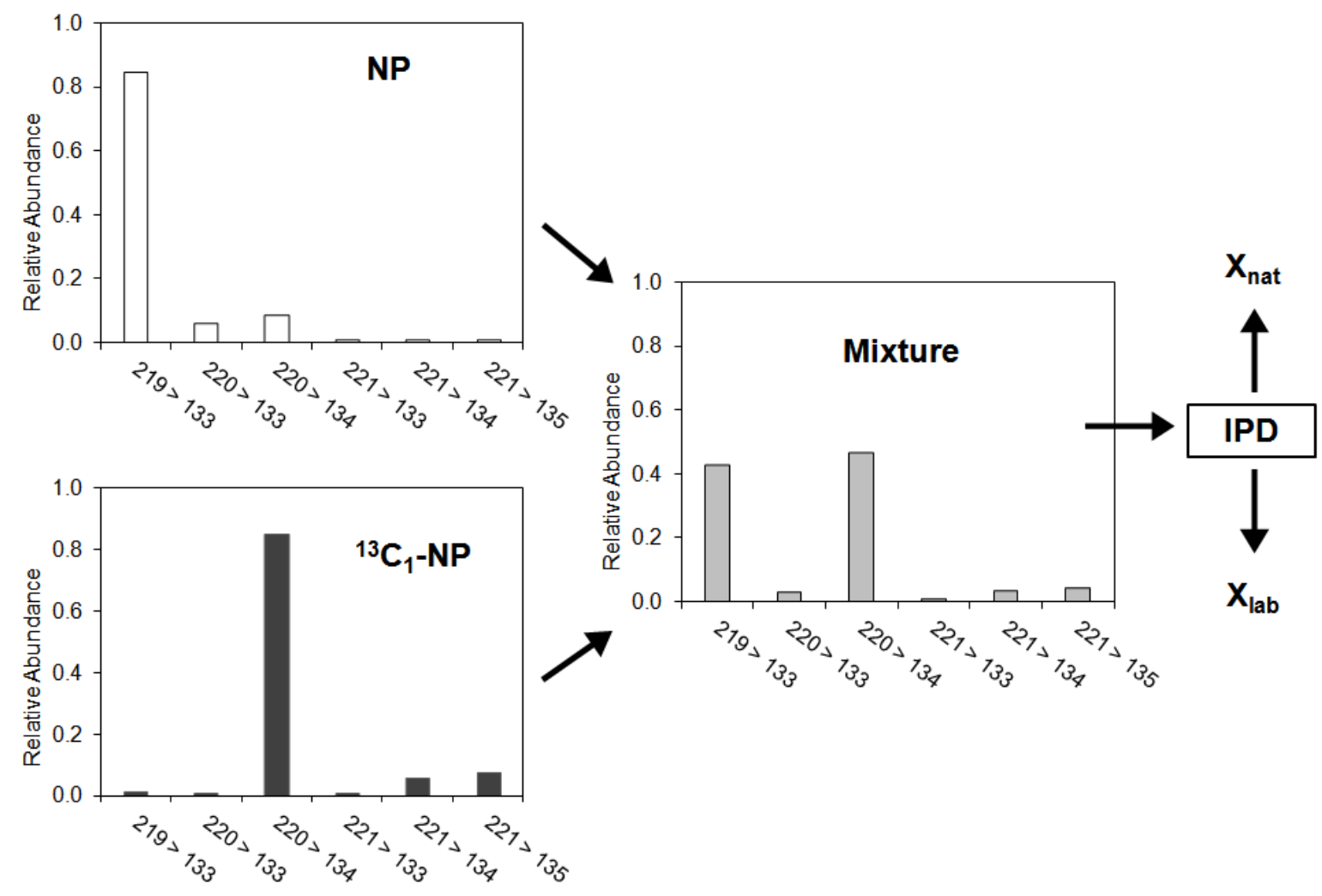

Figure 3. Illustration of the IPD process for a mixture containing an equimolar amount of nonylphenol (NP) and ${ }^{13} \mathrm{C}_{1}$-nonylphenol $\left({ }^{13} \mathrm{C}_{1}\right.$ - NP). 


\section{FIGURE 4}

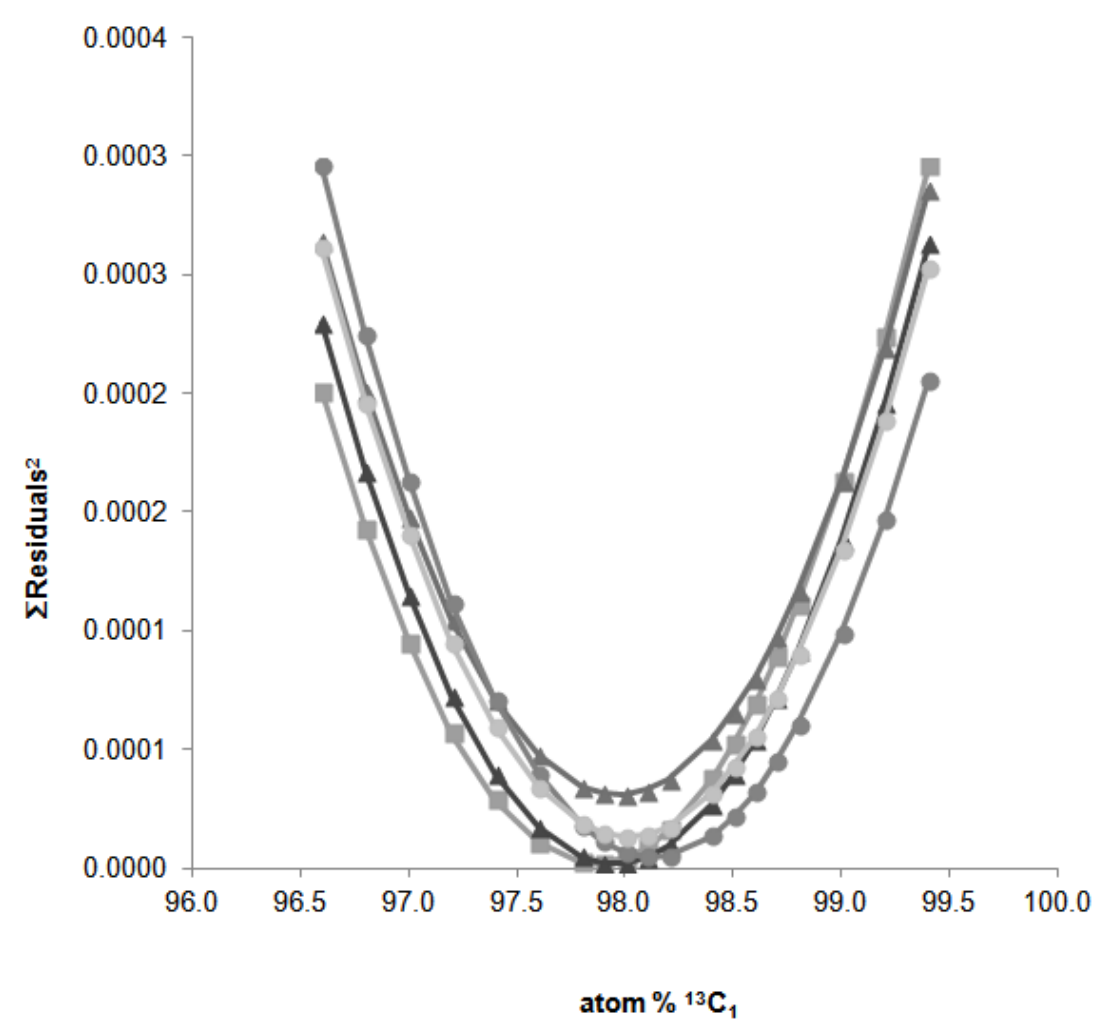

Figure 4. Calculation of the ${ }^{13} \mathrm{C}$ enrichment for a standard solution containing $500 \mathrm{ng} / \mathrm{g}$ of ${ }^{13} \mathrm{C}_{1^{-}}$ nonylphenol $\left({ }^{13} \mathrm{C}_{1}-\mathrm{NP}\right)$. 


\section{FIGURE 5}

a
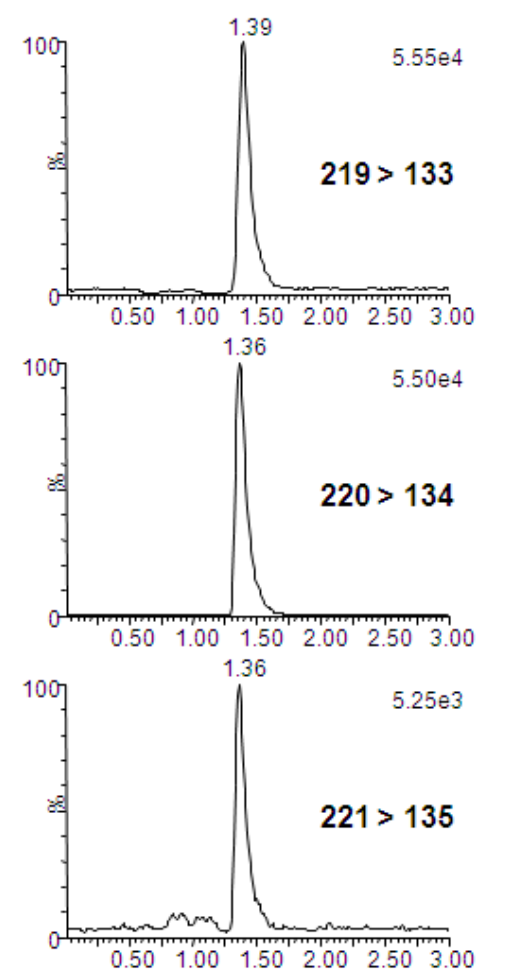

b
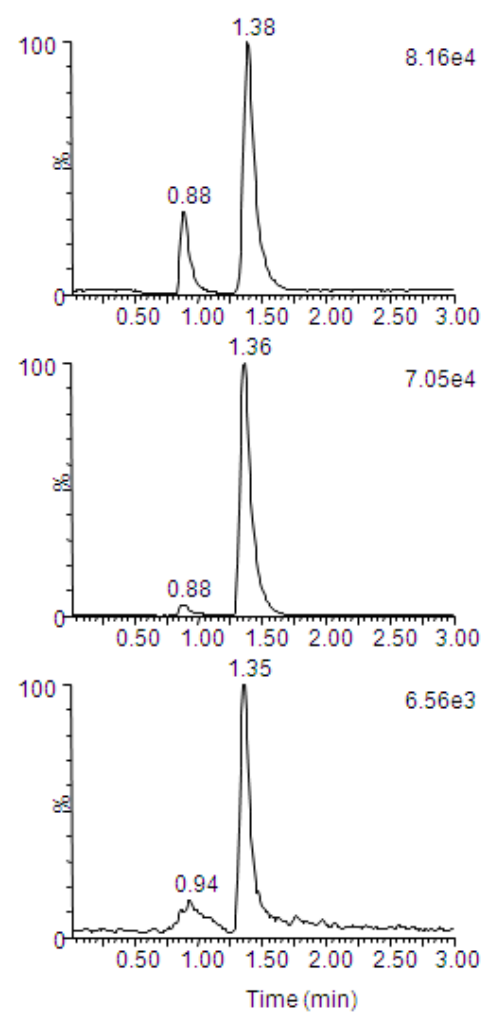

C
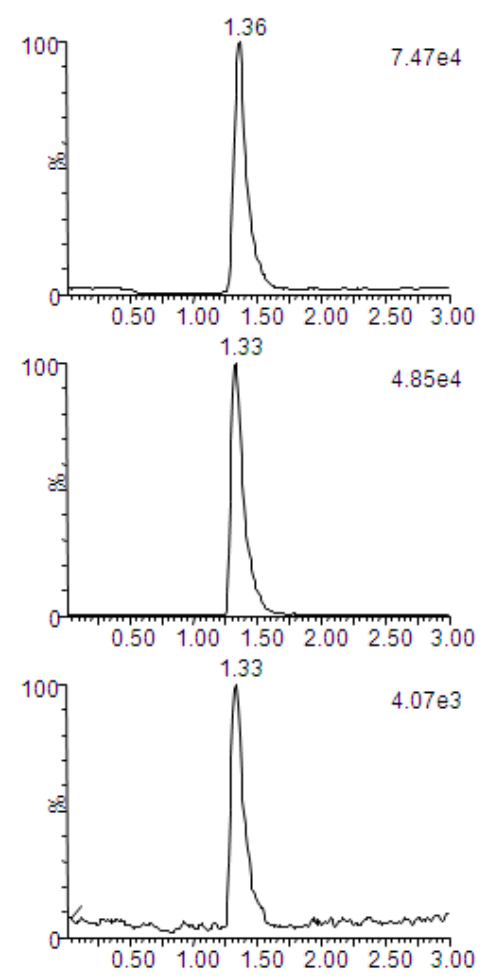

Figure 5. Chromatograms in negative electrospray ionization for technical nonylphenol in; (a) bottled water at $0.1 \mathrm{ng} / \mathrm{g}$, (b) effluent waste water $0.1 \mathrm{ng} / \mathrm{g}$, and (c) influent waste water at $1 \mathrm{ng} / \mathrm{g}$. 


\section{Figure Captions}

Figure 1. Synthesis pathway of labeled 13C1-4-(3,6-Dimethyl-3-heptyl)-phenol(13C1-NP).

Figure 2. GC-EI-MS chromatogram of ${ }^{13} \mathrm{C}_{1}-4-(3,6$-dimethyl-3-heptyl)phenol and mass spectra of a standard of a 4-(3,6-dimethyl-3-heptyl)phenol and $\mathbf{b}^{13} \mathrm{C}_{1}-4$-(3,6-dimethyl-3-heptyl)phenol $\left({ }^{13} \mathrm{C}_{1}-\mathrm{NP}\right)$.

Figure 3. Illustration of the IPD process for a mixture containing an equimolar amount of nonylphenol (NP) and ${ }^{13} \mathrm{C}_{1}$-nonylphenol $\left({ }^{13} \mathrm{C}_{1}\right.$ - NP).

Figure 4. Calculation of the ${ }^{13} \mathrm{C}$ enrichment for a standard solution containing $500 \mathrm{ng} / \mathrm{g}$ of ${ }^{13} \mathrm{C}_{1^{-}}$ nonylphenol $\left({ }^{13} \mathrm{C}_{1}-\mathrm{NP}\right)$.

Figure 5. Chromatograms in negative electrospray ionization for technical nonylphenol in; (a) bottled water at $0.1 \mathrm{ng} / \mathrm{g}$, (b) effluent waste water $0.1 \mathrm{ng} / \mathrm{g}$, and (c) influent waste water at $1 \mathrm{ng} / \mathrm{g}$. 
Table 1. Contribution of the main theoretical pure clusters to the final measured cluster in different water samples for NP

\begin{tabular}{ccccc}
\hline \multirow{2}{*}{ Fragment ion } & \multicolumn{4}{c}{ Relative abundances (\%) } \\
\cline { 2 - 5 } & Bottled water & Surface water & Effluent wastewater & Influent wastewater \\
\hline$\left[\mathrm{M}-\mathrm{H}-\mathrm{C}_{6} \mathrm{H}_{14}\right]^{-}$ & $96.1 \pm 0.4^{\mathrm{a}}$ & $96.2 \pm 0.4$ & $95.4 \pm 0.4$ & $95.5 \pm 0.4$ \\
{$\left[\mathrm{M}-\mathrm{H}-\mathrm{C}_{6} \mathrm{H}_{15}\right]-$} & $3.9 \pm 0.4$ & $3.8 \pm 0.4$ & $4.6 \pm 0.4$ & $4.5 \pm 0.4$
\end{tabular}

${ }^{\mathrm{a}}$ The standard uncertainty of the results corresponds to two UHPLC-MS/MS injections.

Table 2. Recovery of technical nonylphenol in bottled water and effluent and influent waste water obtained by minimal labeling and by a classical calibration curve.

\section{Recovery (\%)}

\begin{tabular}{cccc}
\hline Sample & Validation level (ng/g) & $\begin{array}{c}\text { Minimal labeling and } \\
\text { IPD }\end{array}$ & $\begin{array}{c}\text { Classical calibration } \\
\text { procedure }\end{array}$ \\
\hline Bottled Water & 0.1 & $97 \pm 9^{\mathrm{a}}$ & $165 \pm 8$ \\
& 1 & $104 \pm 5$ & $123 \pm 18$ \\
\hline \multirow{2}{*}{ Effluent Wastewater } & 0.1 & $83 \pm 5$ & $385 \pm 55$ \\
& 1 & $106.0 \pm 1.8$ & $596 \pm 20$ \\
\hline \multirow{2}{*}{ Influent Wastewater } & 1 & $107 \pm 8$ & $157 \pm 41$ \\
& 10 & $108.0 \pm 1.5$ & $151 \pm 55$ \\
\hline
\end{tabular}

${ }^{a}$ The uncertainty of the values corresponds to $1 \mathrm{~s}$ standard deviation of three independent replicates injected three times each.

Table 3. Limits of detection (LODs) and limits of quantification (LOQs) and blank values in the three matrices studied.

\begin{tabular}{cccc}
\hline Sample & $\begin{array}{c}\text { Limit of } \\
\text { Detection } \mathbf{( p g / g )}\end{array}$ & $\begin{array}{c}\text { Limit of } \\
\text { Quantification } \\
\mathbf{( p g / g )}\end{array}$ & $\begin{array}{c}\text { Blank value } \\
\mathbf{( p g / g )}\end{array}$ \\
\hline Bottled water & 9 & 29 & $18.1 \pm 0.6^{\mathrm{a}}$ \\
\hline Effluent Wastewater & 6 & 21 & $102.6 \pm 2.4$ \\
\hline Influent Wastewater & 86 & 290 & $801 \pm 21$ \\
\hline
\end{tabular}

${ }^{a}$ Blank values correspond to one replicate injected three times 